\section{Uso do questionário ISAAC em crianças menores de seis anos: asma ou sibilos transitórios?}

Use of the ISAAC questionnaire in children under six years: asthma or transient wheezing?

Gustavo Silveira Graudenz 1,2

Diogenes Seraphim Ferreira ${ }^{1}$

${ }_{1}$ Departamento de Ciências Médicas, Universidade Nove de Julho, São Paulo, Brasil.

2 Pós-graduação em Gestão Ambiental e Sustentabilidade, Universidade Nove de Julho, São Paulo, Brasil.

\section{Correspondência}

G. S. Graudenz

Departamento de Ciências Médicas, Universidade Nove de Julho.

Rua Vergueiro 235, São Paulo, SP 01504-000, Brasil. graudenz@uninove.br

A asma é uma doença multifatorial, em que a predisposição genética é fortemente influenciada pelas pressões externas. Os achados de Alves et al. 1, em artigo publicado em Cadernos de Saúde Pública, apontam associação entre sintomas de asma e indicadores de exposição à violência comunitária em estudo de base populacional em Salvador, Bahia. Entretanto, outros fatores podem interferir na interpretação dos dados encontrados.

O uso de ferramentas como o questionário padronizado pelo International Study of Asthma and Allergies in Childhood (ISAAC) é prático pela sua facilidade de aplicação em grandes populações, tendo sido validado, no Brasil, para aplicação em escolares de 6-7 anos e adolescentes de 13-14 anos. No entanto, como todo questionário, apresenta limitações quanto aos seus valores preditivos de diagnóstico de asma, especialmente para as crianças mais jovens 2 .

A ocorrência de sibilos na infância está relacionada com infecções virais em parte da faixa etária avaliada (menores de seis anos). Um estudo de corte clássico em asma indicou que a grande maioria das crianças com sibilos até seis anos apresenta condições transitórias associadas com a diminuição das funções de vias aéreas e não tem risco aumentado para desenvolvimento de asma posterior ${ }^{3}$.

Portanto, apesar de a variável resposta "chiados nos últimos 12 meses" ter sido ajustada para outras comorbidades envolvidas no aparecimento de sintomas de asma, não é possível afirmar qual a porcentagem de crianças que sibilaram nos últimos 12 meses e que de fato evoluirão para o quadro de asma brônquica, uma vez que grande parte dos episódios de sibilos na faixa etária inferior a seis anos é autolimitada. Assim sendo, não é adequado falar em prevalência de asma com a metodologia aplicada, e sim em episódios de sibilos, ou sintomas de asma.

Adicionalmente, a persistência desses sibilos está relacionada a outras condições não verificadas nesse estudo. Conforme revisão da literatura, cerca de $80 \%$ dos casos de asma diagnosticados nessa faixa etária são associados a uma reposta mediada por IgE (resposta alérgica) para alguns estímulos imunológicos presentes no ambiente domiciliar, como ácaros, fungos, epitélios de animais ou polens. Os indivíduos com esse tipo de resposta são os que evoluem para a asma brônquica da infância 4 . Conforme indicado pelos autores do artigo publicado em Cadernos de Saúde Pública ${ }^{1}$, exposição à violência comunitária influenciaria o comportamento dos pais, mantendo os filhos por maior tempo em ambientes interiores. Sendo assim, existe a valorização da permanência em casa como fator de risco isolado e a redução do valor individual da exposição à violência como fatores associados ao desenvolvimento de sintomas de asma.

Outro potencial fator de confusão é o tabagismo passivo como determinante de episódios de sibilos recorrentes 4,5. Esse fator pode estar inter-relacionado com a violência e a condição socioeconômica da população avaliada.

Uma possibilidade de avançar com o estudo realizado seria estratificar a faixa etária dos adolescentes (12 anos) como forma de melhorar o valor preditivo do questionário, ou mesmo realizar um estudo casocontrole, dividindo a população de acordo com seu grau de exposição à violência, controlando as diferenças de sensibilização a aeroalérgenos e exposição ao tabagismo passivo. Idealmente, devem-se realizar espirometrias para demonstrar a obstrução de vias aéreas reversível, esta, sim, conclusiva para diagnósticos de asma, de modo a confirmar os achados relativos ao papel da violência comunitária e prevalência de asma brônquica.

\section{Colaboradores}

G. S. Graudenz e D. S. Ferreira contribuíram com análise do artigo, redação e correção do texto.

1. Alves GC, Santos DN, Feitosa CA, Barreto ML. Community violence and childhood asthma prevalence in peripheral neighborhoods in Salvador, Bahia State, Brazil. Cad Saúde Pública 2012; 28:86-94. 
2. Wandalsen NF, Gonzalez C, Wandalsen GF, Solé D. Evaluation of criteria for the diagnosis of asthma using an epidemiological questionnaire. J Bras Pneumol 2009; 35:199-205.

3. Martinez FD, Wright AL, Taussig LM, Holberg CJ, Halonen M, Morgan WJ, et al. Asthma and wheezing in the first six years of life. The Group Health Medical Associates. N Engl J Med 1995; 332:133-8.

4. Wright AL. Epidemiology of asthma and recurrent wheeze in childhood. Clin Rev Allergy Immunol 2002; 22:33-44.

5. Palvo F, Toledo EC, Menin AMCR, Jorge PPO, Godoy MF, Solé D. Risk factors of childhood asthma in Sao Jose do Rio Preto, Sao Paulo, Brazil. J Trop Pediatr 2008; 54:253-7.

Recebido em 22/Fev/2012

Aprovado em 18/Abr/2012

Alves $\mathrm{GC}$ et al. respondem

Alves GC et al. respond

Guilherme da Costa Alves 1

Darci Neves Santos 1

Caroline Alves Feitosa ${ }^{1}$

Mauricio Lima Barreto 1

${ }_{1}$ Instituto de Saúde Coletiva, Universidade Federal da Bahia, Salvador, Brasil.

\section{Correspondência}

G. C. Alves

Instituto de Saúde Coletiva, Universidade Federal da Bahia.

Rua Basílio da Gama s/n, Campus Universitário do Canela, Salvador, BA 40110-040, Brasil. guilhermec.ufba@gmail.com

Graudenz \& Ferreira estão corretos em afirmar que seria mais apropriado empregar o termo prevalência de sintomas de asma ao invés de prevalência de asma. Denominando-a dessa forma, os sintomas relatados podem não significar necessariamente diagnóstico de asma. Porém, o uso de sibilo nos últimos 12 meses como definição de asma, é uma das formas bem aceitas e generalizadas em estudos epi- demiológicos, pela simplicidade e comparabilidade de dados entre distintos países 1,2 . Sobre IgE como mediador da relação entre violência comunitária e asma, não somos defensores desta hipótese. Estudos realizados no Brasil e Equador encontraram que a maior parte da prevalência de asma não foi explicada por uma resposta alérgico-atópica, quer seja pela presença de IgE, ou positividade do teste cutâneo ${ }^{3}$. Em Salvador, somente 20\% da asma foi atribuível à alergia 4 , achado similar àqueles procedentes de outros países em desenvolvimento 5 . Ademais, outras evidências produzidas por nosso grupo, na abordagem do papel de outros fatores psicossociais, também sugerem um efeito independente do estado atópico 6,7. Por outro lado, é do nosso interesse explorar a influência da violência comunitária separadamente para asma atópica e não atópica, sendo este um dos objetivos de um futuro estudo.

A influência do tabagismo passivo, apesar de ser um fator clássico relacionado à ocorrência de asma, também foi avaliada nessa mesma população ${ }^{8}$, sem evidência de associação com os sintomas de asma.

Em que pese a possibilidade de melhoria da qualidade dos resultados pela estratificação da faixa etária dos adolescentes, a existência de apenas uma criança com 12 anos em nossa amostra (e de 37 acima de 11) inviabilizou análises mais segmentadas.

Ressaltamos ainda que três argumentos embasaram a plausibilidade da relação entre violência comunitária e asma. Primeiro, o comportamento dos pais, seguido do acesso prejudicado aos serviços de saúde e da ação do estresse como fator mais proximal e individual nessa relação ${ }^{9}$. Consideramos tais argumentos como possíveis mecanismos igualmente relevantes para explicar a associação entre violência comunitária e asma, não sendo prudente priorizar algum em detrimento dos demais.

Para futuros estudos é bastante apropriada a indicação do desenho caso-controle a fim de aprofundar a compreensão dos efeitos da violência. Agradecemos por sugerir o uso da espirometria, principalmente porque o SCAALA possui essa informação a ser explorada em estudo próximo.

Por fim, destacamos que no Brasil é recente a investigação da relação entre fatores psicossociais e asma, apresentando ainda algumas limitações. Por outro lado, tem contribuído substancialmente na construção de novos modelos que possam dar conta do complexo processo de multideterminação da asma na América Latina 3,6,7,8.

1. The International Study of Asthma and Allergies in Childhood (ISAAC) Steering Committee. Worldwide variation in prevalence of symptoms of asthma, allergic rhinoconjunctivitis, and atopic eczema: ISAAC. Lancet 1998; 351:1225-32. 
2. Pearce N, Aït-Khaled N, Beasley R, Mallol J, Keil U, Mitchell E, et al. Worldwide trends in the prevalence of asthma symptoms: phase III of the International Study of Asthma and Allergies in Childhood (ISAAC). Thorax 2007; 62:758-66.

3. Cooper PJ, Rodrigues LC, Barreto ML. Influence of poverty and infection on asthma in Latin America. Curr Opin Allergy Clin Immunol 2012; 12:171-8.

4. Cunha SS, Barreto ML, Fiaccone RL, Cooper PJ, Alcantara-Neves MN, Simões SM, et al. Asthma cases in childhood attributed to atopy in tropical Area in Brazil. Rev Panam Salud Pública 2010; 28:405-11.

5. Weinmayr G, Weiland SK, Björsktén B, Brunekreef B, Büchele G, Cookson WO, et al. Atopic sensitization and the international variation of asthma symptom prevalence in children. Am J Respir Crit Care Med 2007; 176:565-74

6. Barreto-do-Carmo MB, Santos DN, Amorim LDAF, Fiaccone RL, Cunha SS, Rodrigues LC, et al. Minor psychiatric disorders in mothers and asthma in children. Soc Psychiatry Psychiatric Epidemiology 2009; 44:416-20.
7. Santos LM, Santos DN, Rodrigues LC, Barreto ML. Maternal mental health and social support: effect on childhood atopic and non-atopic asthma symptoms. J Epidemiol Community Health 2012; [Epub ahead of print].

8. Feitosa CA, Santos DN, Barreto-do-Carmo MB, Santos LM, Teles CA, Rodrigues LC, et al. Behavior problems and prevalence of asthma symsptoms among Brazilian children. J Psychosom Res 2011; 71:160-5.

9. Alves GC, Santos DN, Feitosa CA, Barreto ML. Community violence and childhood asthma prevalence in peripheral neighborhoods in Salvador, Bahia State, Brazil. Cad Saúde Pública 2012; 28:86-94.

Recebido em 18/Mai/2012

Aprovado em 25/Mai/2012 